\title{
Comparisons of Single Versus Multiple Bacterial Species on Biological Control of Anthurium Blight
}

\author{
R. Fukui, H. Fukui, and A. M. Alvarez
}

Department of Plant Pathology, University of Hawaii at Manoa, Honolulu 96822-2279.

Accepted for publication 16 January 1999.

\begin{abstract}
Fukui, R., Fukui, H., and Alvarez, A. M. 1999. Comparisons of single versus multiple bacterial species on biological control of anthurium blight. Phytopathology 89:366-373.

Effects of single versus multiple biological control agents (BCAs) on suppression of bacterial blight of anthurium were studied using a bioluminescent strain (V108LRUH1) of Xanthomonas campestris pv. dieffenbachiae. When five BCAs (GUT3, GUT4, GUT5, GUT6, and GUT9) were coinoculated in various combinations with V108LRUH1 into filter-sterilized guttation fluids of anthurium plants, a mixture of all five strains or four strains without GUT9 was most inhibitory to V108LRUH1. None of the individual BCAs inhibited V108LRUH1 in the guttation fluid. When BCAs were sprayed at $\cong 10^{8} \mathrm{CFU} / \mathrm{ml}$ on the foliage of a susceptible culti-

var 1 day prior to inoculation with V108LRUH1, GUT6 alone and any mixtures containing GUT6 were highly effective in suppressing wound invasion and subsequent leaf infection by V108LRUH1. When tested on several cultivars that differed in susceptibility to the disease, the mixture of five strains or four strains without GUT9 consistently suppressed leaf infection regardless of the cultivars. In some cultivars, BCAs completely suppressed both wound and hydathode invasion by V108LRUH1, resulting in no infection in many leaves. These results indicate that application of bacterial mixtures provides anthurium cultivars with bacterial communities suppressive to $X$. campestris pv. dieffenbachiae. The results also suggest that selecting an effective mixture of BCAs first and then removing ineffective strains may be a better general approach to finding the most effective BCAs than finding individual strains and combining them.
\end{abstract}

Bacterial blight of anthurium (Anthurium andraeanum Lind. ex André), caused by Xanthomonas campestris pv. dieffenbachiae, is a difficult disease to control because of the inoculum persisting in the commercial field in the form of symptomless infections $(4,17)$ and the ease of transmission in areas of high precipitation, such as Hawaii. Anthurium, as a long-term perennial crop (up to 9 years), is continuously threatened by the disease. Currently, no effective pesticides are available for bacterial blight in Hawaii. Tolerant cultivars are available (10-12), but susceptible cultivars are widely grown because of the market demand for their desirable flower shapes and colors. The disease occurs throughout the Pacific-Caribbean basins and recently has been found among propagative materials shipped to India from the Netherlands (21). Production of pathogen-free plants by in vitro propagation followed by strict sanitation measures helps reduce the disease, but does not eliminate the problem $(15,17)$. A new approach for disease control is needed to ensure adequate production.

Promising candidates for biological control agents (five different bacterial species) were isolated from the guttation fluids of anthurium plants (3). A mixture of five species was highly inhibitory to $X$. campestris pv. dieffenbachiae when coinoculated in the filtersterilized guttation fluid. However, none of the individual strains were inhibitory to the pathogen. Such a case was reported earlier for biological control of take-all of wheat; a mixture of five strains of fluorescent pseudomonads suppressed take-all in raw soil, but the individual strains were not effective (25). Many other reports indicated that combination of antagonistic bacterial strains resulted in improved biological control in various bacterial and fungal diseases $(7,8,14,19,20,22)$. In contrast, Fukui et al. (5) reported that the use of multiple bacterial strains was not superior to the use of the same individual strains for controlling pericarp infection by

Corresponding author: A. M. Alvarez; E-mail address: alvarez@hawaii.edu

Publication no. P-1999-0304-01R

(C) 1999 The American Phytopathological Society
Pythium spp. In this case, multiple fluorescent Pseudomonas strains introduced to the same ecological niche (spermosphere of sugar beet) competed with or inhibited each other as they interacted with Pythium spp., resulting in no enhancement or even reduced efficacy in biological control.

Population studies of the biocontrol agents (BCAs) and X. campestris pv. dieffenbachiae in the guttation fluid provided no indication of antagonistic relationships among the five BCAs. The five bacterial species all survived in the guttation fluid for 2 weeks, while only the pathogen population diminished (3). When sprayed onto anthurium leaves, the mixture of five species was highly effective in preventing wound invasion and subsequent leaf infection by $X$. campestris pv. dieffenbachiae. However, effects of the individual BCAs on disease suppression have not been compared systematically with the effect of the mixture in planta. Such a study is important for understanding interactions among BCAs and the roles of multiple BCAs in suppression of anthurium blight.

The objectives in this study were to determine the effects of individual strains and various combinations of BCAs on suppression of leaf infection by $X$. campestris pv. dieffenbachiae and to characterize their efficacy in biological control on several anthurium cultivars that differed in susceptibility to blight. A bioluminescent strain of $X$. campestris pv. dieffenbachiae (2) was used to monitor the progression of leaf infection and determine disease severity.

\section{MATERIALS AND METHODS}

Pathogen and BCAs. Bioluminescent strain V108LRUH1 of $X$. campestris pv. dieffenbachiae, containing a transposon sequence Tn4431 (24), was used in this study (2). The presence of Tn4431 in this strain was confirmed by colony growth on 523 medium (9) containing rifampicin and tetracycline (50 and $10 \mu \mathrm{g} / \mathrm{ml}$, respectively) and bioluminescence emission from the colonies.

Five BCAs, designated GUT3, GUT4, GUT5, GUT6, and GUT9, originally were isolated from guttation fluids of two susceptible anthurium cultivars (GUT3, GUT4, and GUT5 from Marian Seefurth; 
GUT6 and GUT9 from UH1060) (3). Identities of the five BCAs were determined by the standard bacteriological tests $(13,26)$, fatty acid analysis, API-NFT rapid identification system (bioMérieux Vitek, Inc., Hazelwood, MO), and Biolog MicroStation system (Biolog, Inc., Hayward, CA) with the database MicroLog3 (release 3.50; Biolog, Inc.) using the standard BUGM medium with 5\% sheep blood. Species identification by fatty acid analysis was done through the courtesy of R. Stall, Plant Pathology Department, Institute of Food and Agricultural Sciences, University of Florida.

Peptone glucose medium $(1.0 \%$ peptone, $0.5 \%$ glucose, and $1.7 \%$ agar) was used to grow V108LRUH1 for inoculum preparation. Yeast extract-dextrose-calcium carbonate (YDC) medium (27) was used to grow strains of BCAs for inoculum preparation.

Plant materials and growth conditions. Seven cultivars of anthurium were obtained from growers on the island of Hawaii: Alii (UH908), ARCS (UH1068), Kalapana (UH1016), Marian Seefurth (H33), Rainbow (no UH number), Tropic Mist (UH780), and UH1060 (no common name). 'Marian Seefurth', 'Rainbow', and 'UH1060' are susceptible to foliar infection, and the rest are tolerant or less susceptible as judged by susceptibility evaluation using the bioluminescent strain V108LRUH1 (4). Plants were repotted in cinder in $10 \times 10-\mathrm{cm}$ pots and fertilized with Nutricote (Chisso Asahi Co., Ltd., Tokyo) 13-13-13 plus microelements in a 70-day release formulation at a rate of approximately 0.6 to $0.7 \mathrm{~g}$ of fertilizer per pot. The plants were grown (acclimatized) in a glasshouse with shading provided by two layers of saran sheet $(2,4)$. The daily minimum and maximum temperatures in the glasshouse were recorded.

Growth and survival of V108LRUH1 in the guttation fluid coinoculated with various combinations of BCAs. To collect guttation fluid, the two youngest leaves of individual 'Marian Seefurth' plants (10 to 20 plants for each collection) were disinfested by wiping with $70 \%$ ethanol and covered in the evening with a clean plastic bag. Plants were then watered by drenching. The next morning, guttation fluids that accumulated inside the bags and retained on the leaves were collected, pooled, and filter-sterilized (pore size $=0.2 \mu \mathrm{m}$, Supor Acrodisc 25; Gelman Sciences, Ann Arbor, MI). Cells of V108LRUH1 grown on peptone dextrose medium for 2 days were suspended in sterile $10 \mathrm{mM}$ phosphate buffer ( $\mathrm{pH}$ 6.9) to achieve a cell density of 2.0 to $3.0 \times 10^{8} \mathrm{CFU} / \mathrm{ml}$, and $15 \mu \mathrm{l}$ of this cell suspension was inoculated into $1.47 \mathrm{ml}$ of filtered guttation fluid in a sterile tube $(100 \times 13 \mathrm{~mm})$. Cells of five BCAs grown on plates of YDC medium for 2 days were individually suspended in sterile phosphate buffer to achieve cell densities of 1.0 to $3.0 \times 10^{8} \mathrm{CFU} / \mathrm{ml}$, and the five cell suspensions were mixed with equal volumes in various combinations. The combinations of BCAs tested were a mixture of all five BCAs (GUT3 plus GUT4 plus GUT5 plus GUT6 plus GUT9, hereafter described as mixture 34569), all four-strain combinations (mixtures 4569, 3569, 3469, 3459, and 3456), several three-strain combinations (mixtures 456, 356, 346, 345, 369, 469, and 569), and all two-strain combinations with GUT6 (mixtures $36,46,56$, and 69 ). A total of $15 \mu \mathrm{l}$ of each blended cell suspension was inoculated to the guttation fluid samples (one tube per combination). Filter-sterilized guttation fluid not inoculated with any BCAs was used for the control. The tubes were covered with caps, sealed with Parafilm, and incubated without shaking at $28^{\circ} \mathrm{C}$. After incubation for 7 and 14 days, the cell density of V108LRUH1 was determined by removing $100 \mu \mathrm{l}$ of guttation fluid for dilution plate counting on peptone dextrose medium supplemented with rifampicin, tetracycline, and cycloheximide (at 50, 10, and $100 \mu \mathrm{g} / \mathrm{ml}$, respectively). The test was conducted twice using guttation fluid of 'Marian Seefurth', and the two tests were considered as two replicates. The incubation period was considered as the repeated measure. The initial inoculum density of V108LRUH1 was estimated by inoculating $15 \mu \mathrm{l}$ of the cell suspension into $1.485 \mathrm{ml}$ of sterile phosphate buffer (six replicates), followed by dilution counting.

A similar test was conducted using guttation fluids collected from three different cultivars: Marian Seefurth, Kalapana, and Rainbow. The strain combinations tested were mixtures 34569, 4569,
3569, 3469, 3459, 3456, 456, 356, 346, 345, 56, 46, 45, and five individual strains. The procedure was the same as described above. Cultivars were considered as replicates because the growth and survival of V108LRUH1 in filter-sterilized guttation fluids of different cultivars was not significantly different (3). In this test, cell densities of individual BCAs in the cell suspensions (before mixing) were determined by dilution plate counting on Kelman's triphenyl tetrazoliumchloride medium modified by Norman and Alvarez (16). The test was done twice.

Effects of individual and multiple BCAs on suppression of wound invasion and subsequent leaf infection by V108LRUH1. The test was conducted with 'Marian Seefurth' plants. Before inoculation, the surfaces of the two youngest leaves in each plant were disinfested by wiping with $70 \%$ ethanol and the plants were placed inside clean plastic bags. Cells of five BCAs grown on YDC plates for 2 days were suspended individually in sterile distilled water to achieve an optical density (at $600 \mathrm{~nm}$ ) of 0.2 (cell densities at 1.0 to $3.0 \times 10^{8} \mathrm{CFU} / \mathrm{ml}$ ) and diluted twofold with sterile distilled water. Plants were spray-inoculated with either cell suspensions of individual BCAs or three different blended suspensions (mixed at equal volumes): 34569,345 , and 69. Control plants were sprayed with sterile distilled water. All plants were kept in sealed plastic bags for $4 \mathrm{~h}$ and removed from the bags in the glasshouse at night to allow the leaves to dry. The next morning, the two youngest leaves on each plant were clipped at four marginal sites ( $\cong 5 \mathrm{~mm}$ deep) to create wounds. Cell suspensions of BCAs (individual and blended) were prepared at 1.0 to $3.0 \times 10^{8} \mathrm{CFU} / \mathrm{ml}$ as described above, and one drop of the cell suspension (sterile distilled water for control) was inoculated directly to each wound by delivering the suspensions with a pipette. After the leaves dried, whole plants were spray-inoculated with a cell suspension of V108LRUH1 (at $\cong 2.0 \times$ $10^{6} \mathrm{CFU} / \mathrm{ml}$ ), and kept wet overnight in the plastic bags at room temperature $\left(22 \pm 1^{\circ} \mathrm{C}\right)$. The next day, plants were removed from the bags and arranged in a randomized complete block (RCB) design with five blocks (five replicates per treatment) in the glasshouse. Plants were watered without wetting the leaves every other day, and severity of leaf infection was determined 19, 27, and 40 days after inoculation by recording bioluminescence images on X-ray film as described previously (4). Disease was assessed on two clipped leaves on each plant (10 observations per treatment), because the severity of leaf infection was greatest in the two youngest leaves among leaves of all ages and was not statistically different between the two youngest leaves (4). The average minimum and maximum temperatures ( \pm 1 standard deviation) during the experiment were $24.4 \pm 0.6$ and $32.9 \pm 1.2^{\circ} \mathrm{C}$, respectively.

This experiment was conducted again to test the effects of five individual BCAs and several other combinations of BCAs. The combinations tested were mixtures 34569, 3456, 456, 3469, 469, and 69. Plants of 'Marian Seefurth' were inoculated with BCAs and V108LRUH1 as described above and arranged in a RCB design with five blocks in the glasshouse. Severity of leaf infection was determined on two leaves in each plant (10 observations per treatment) 18,28 , and 43 days after inoculation. The average minimum and maximum temperatures during the experiment were $21.6 \pm 1.7$ and $34.7 \pm 1.5^{\circ} \mathrm{C}$, respectively.

Effects of blended BCAs on suppression of wound invasion and subsequent leaf infection by V108LRUH1 in various cultvars. The effects of mixtures 34569 and 3456 that were most efficacious on 'Marian Seefurth' plants in suppression of wound invasion were compared among five different cultivars: Alii, ARCS, Kalapana, Marian Seefurth, and UH1060. Leaves were disinfested with $70 \%$ ethanol and clipped (two youngest leaves per plant) prior to inoculation as described above. Cell suspensions of BCAs were prepared as described for the previous plant inoculation tests and blended (at equal volumes) to make two different strain mixtures. Plants were inoculated with BCAs (or sterile distilled water for the controls) and V108LRUH1 as described previously. Treatments were arranged in a RCB design with six blocks (six replicates per 
treatment). Severity of leaf infection was determined on two leaves in each plant (12 observations per treatment) 20,31, 39, and 55 days after inoculation. The average minimum and maximum temperatures during the experiment were $21.1 \pm 1.5$ and $28.3 \pm 1.7^{\circ} \mathrm{C}$, respectively.

In a separate trial, the effects of mixture 34569 and two individual strains (GUT5 and GUT6) on suppression of wound infection were compared on 'ARCS', 'Kalapana', 'Marian Seefurth', and 'Tropic Mist'. GUT5 and GUT6 were the least and most effective strains, respectively, among five individual BCAs tested on 'Marian Seefurth'. Plant were treated with a cell suspension of) and then inoculated with either GUT5, GUT6, mixture 34569, or sterile distilled water (control V108LRUH1 as described above. Treatments were arranged in a RCB design with six blocks. Severity of leaf infection was determined on two leaves of each plant (12 observations per treatment) 20,32, and 43 days after inoculation. The average minimum and maximum temperatures during the experiment were $23.6 \pm 0.8$ and $30.9 \pm 1.6^{\circ} \mathrm{C}$, respectively.

Statistical analysis. For the in vitro tests using filter-sterilized guttation fluid, strain mixture was the main effect and incubation time was the repeated measures factor. Cultivars were considered as replicates. For plant inoculation tests, both strain and cultivar were considered as the main effects. Disease assessment day was the repeated measures factor in a factorial arrangement. Severity of leaf infection was assessed by three examiners. Mean values (percentage data) were first transformed by the arcsine transformation, and transformed data were analyzed by analysis of variance. Means were separated by Fisher's least significant difference test or Student-Newman-Keuls test.

Severity of leaf infection was expressed as a disease severity index (an average percentage in 10 or 12 observations). Thus, a value of disease severity index corresponds to a percentage of infected leaf area.

\section{RESULTS}

Identification of BCAs. Test results are summarized in Table 1. GUT3 and GUT5 were identified as Sphingomonas paucimobilis (formerly Pseudomonas paucimobilis) (30) and Brevundimonas vesicularis (formerly $P$. vesicularis) (23), respectively, as determined by at least two of the three different tests. GUT4 was identified as a Microbacterium sp. by two tests. A definitive identification was not obtained for GUT6 by the three tests, but it is probably a nonfluorescent Pseudomonas sp. The closest species to GUT9 was Alcaligenes eutrophus or $P$. stutzeri. The nutrient utilization profiles of the five BCAs and V108LRUH1 determined by the Biolog System (using Biolog GN microplates for all strains) revealed substantial difference in carbon utilization patterns among the strains of BCAs. Of 39 carbon sources that are utilized by V108LRUH1, 37 sources are also utilized by at least one of five BCAs.

Growth and survival of V108LRUH1 in the guttation fluid coinoculated with various combinations of BCAs. The mixture consisting of all five BCAs significantly $(P=0.01)$ reduced the cell density of V108LRUH1 (by approximately $10^{3}$-fold) during 14 days of incubation. This inhibitory effect of BCAs on growth of V108LRUH1 was diminished or nearly nullified when certain strains were removed from the mixture (Fig. 1). Removal of GUT5 (3469) nearly nullified the inhibitory effect of the bacterial mixture. Removal of GUT4 (3569) also diminished the inhibitory effect. However, the four-strain mixture missing either GUT3 (4569), GUT6 (3459), or GUT9 (3456) had a significant inhibitory effect on V108LRUH1. Further removal of GUT3 or GUT4 from mixture 3456 did not significantly diminish inhibitory effect of the bacterial mixture, but removal of GUT5 or GUT6 resulted in a significant reduction in the inhibitory effect. Other mixtures $(36,46,56,69,369,469$, and 569) did not have significant inhibitory effects on V108LRUH1.

When BCAs in various combinations were tested against V108LRUH1 in guttation fluids of three cultivars, mixture 3456 was the most inhibitory, followed by mixture 34569 (Fig. 2). Other four-strain mixtures were significantly less inhibitory than mixture 3456. Mixture 3569 was among the least inhibitory strain combinations. Mixtures consisting of two or three strains were significantly less inhibitory than 3456, and those devoid of GUT4 (56 and 356) or both GUT3 and GUT5 (46) did not have significant inhibitory effects on V108LRUH1. None of the BCAs exhibited inhibitory effects when tested individually. Inhibitory effects of various strain mixtures on V108LRUH1 varied slightly depending on the cultivars from which guttation fluids were collected. However, the overall trend was similar among three guttation fluids.

Effects of BCAs on leaf infection from wounds. When BCAs were applied individually to 'Marian Seefurth' plants, GUT6 was most effective in preventing wound invasion and suppressing subsequent leaf infection by V108LRUH1 (Fig. 3). GUT4 and GUT5 applied alone were least effective in both tests. All mixtures of BCAs containing GUT6 (including mixtures 34569 and 3456) were highly effective in preventing wound invasion, but their overall effects on suppression of leaf infection were not greater $(P=0.01)$ than the effect achieved by GUT6 alone. Mixture 345 was significantly less effective than mixture 34569 .

Effects of BCAs on leaf infection in various cultivars. Mixtures 34569 and 3456 were highly effective in suppressing leaf infection in five anthurium cultivars, regardless of their susceptibility to blight. Both mixtures were equally effective in preventing wound invasion in all five cultivars (Fig. 4A), and disease severity for the plants treated with mixture 3456 (39 days after inoculation) was not significantly different from that for the plants treated with mixture 34569 in all cultivars (Fig. 4B). When disease severity for BCA-treated plants was compared 55 days after inoculation, disease severity for 'Marian Seefurth' was significantly higher than those for four other cultivars (data not shown). This was because infection initiated from the hydathodes (but not from the wounds) of 'Marian Seefurth' became evident in the late stage of disease evaluation. Disease severity for control plants evaluated 39 days after inoculation was highest for 'UH1060' and 'ARCS', followed by

TABLE 1. Identification of biocontrol agents

\begin{tabular}{|c|c|c|c|}
\hline Strain & Biolog system $^{\mathrm{a}}$ & Fatty acids analysis & API NFT system \\
\hline GUT3 & Sphingomonas paucimobilis $(0.561)^{\mathrm{b}, \mathrm{c}}$ & Sphingomonas capsulata $(0.352)^{\mathrm{d}}$ & Sphingomonas paucimobilis \\
\hline GUT4 & Microbacterium $\mathrm{sp} .(0.806)^{\mathrm{e}}$ & Microbacterium imperiale $(0.427)$ & No ID I $^{\mathrm{f}}$ \\
\hline GUT5 & Brevundimonas vesicularis $(0.768)^{\mathrm{g}}$ & Brevundimonas vesicularis (0.613) & Brevundimonas vesicularis \\
\hline GUT6 & Nonfluorescent Pseudomonas sp. $(0.496)^{\mathrm{h}}$ & Pseudomonas rubrisubalbicans (0.757) & No ID \\
\hline GUT9 & Alcaligenes eutrophus (0.596) & Pseudomonas stutzeri $(0.271)$ & No ID \\
\hline
\end{tabular}

a According to the database of MicroLog3 (release 3.50), using the standard BUGM medium with 5\% sheep blood.

${ }^{\mathrm{b}}$ S. paucimobilis Group A, according to identification by the Biolog system. S. paucimobilis $=$ P. paucimobilis (27).

c Similarity index by the Biolog system.

${ }^{\mathrm{d}}$ Similarity index by fatty acid analysis.

${ }^{\mathrm{e}} \mathrm{CDC}$ group A-4 (Microbacterium sp.), according to identification by the Biolog system.

${ }^{\mathrm{f}}$ Identification was not achieved by the API NFT system.

$\mathrm{g} B$. vesicularis $=P$. vesicularis $(20)$.

${ }^{\text {h }}$ Pseudomonas-like group 2, according to identification by the Biolog system. 
'Marian Seefurth', and least for 'Kalapana' and 'Alii' (Fig. 4B). Data for 20 and 31 days after inoculation were not presented, because differences were more evident in the late stage of disease development. In the control plants, most of the wounds were invaded by the pathogen in all cultivars ( 43 of 48 clipped sites in total) with the exception of 'Alii' (32 of 48 sites) (Fig. 4A).

When GUT5 and GUT6 were tested individually on four different cultivars, GUT6 was nearly as effective as mixture 34569 in suppressing leaf infection. Both GUT6 and mixture 34569 were highly effective in preventing wound invasion, regardless of the cultivars (Fig. 4C). In three cultivars (Kalapana, Marian Seefurth, and Tropic Mist), the effect of GUT6 on disease suppression was not significantly different from the effect of the mixture (Fig. 4D). In 'ARCS', however, the effect by the mixture on disease suppression was greater $(P=0.01)$ than the effect by GUT6. As observed in the previous test, disease severity on the plants treated with the mixture was not significantly different among the cultivars evaluated 32 days after inoculation (data not shown). By 43 days after inoculation, however, disease severity on the treated plants of 'Marian Seefurth' was significantly greater than those of the other three cultivars (Fig. 4D). In contrast to GUT6, GUT5 was considerably less effective in suppressing leaf infection and wound invasion by V108LRUH1 than mixture 34569 in 'Marian Seefurth', 'Tropic Mist', and 'Kalapana', but was as effective as the mixture in 'ARCS' (Fig. 4C and D). Data for 20 and 32 days after inoculation were not presented, because differences were more evident in the late stages of disease development. For the plants inoculated only with V108LRUH1, there were significant differences in disease severity among the four cultivars, but not in the number of wound invasions.

In these two tests (Fig. 4), both wound and hydathode invasions by V108LRUH1 were completely suppressed in many BCA-treated leaves, resulting in no infection. Such cases of noninfection were observed often in 'Alii', 'ARCS', and 'UH1060' (5 to 11 of 12 as-

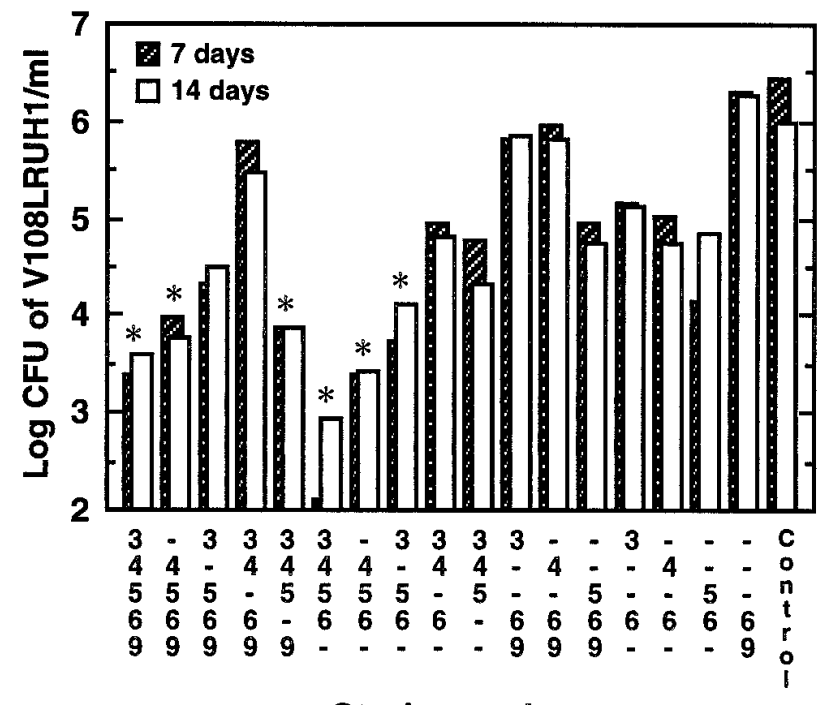

\section{Strain number}

Fig. 1. Survival of V108LRUH1 in filter-sterilized guttation fluid of 'Marian Seefurth' plants coinoculated with various combinations of biocontrol agents (BCAs). The numbers 3, 4, 5, 6, and 9 represent strains GUT3, GUT4, GUT5, GUT6, and GUT9, respectively. The test was conducted twice with one replicate, and two tests were considered as two replicates. Cell density of V108LRUH1 was determined 7 and 14 days after inoculation. According to two-way (treatment and time) analysis of variance, the effect of treatment (combinations of the BCAs) was significant at $P<0.001$ and the effect of time (incubation period) and the treatment $\times$ time interaction were not significant $(P>0.05)$. Values for 14 days after inoculation, indicated with asterisks, were significantly different $(P=0.01)$ from the value for control according to Fisher's least significant difference test. The initial cell density of V108LRUH1 was 6.6 $\mathrm{CFU} / \mathrm{ml}$ in both tests. sessed leaves), occasionally in 'Kalapana' and 'Tropic Mist' (1 to 2 of 12 leaves), but never in 'Marian Seefurth'.

\section{DISCUSSION}

Application of mixtures of BCAs established inhibitory bacterial communities on anthurium cultivars that was superior to individual strains in achieving effective and consistent biocontrol of bacterial blight over a range of anthurium cultivars. Interaction between V108LRUH1 and the BCAs in guttation fluids revealed that inhibition of V108LRUH1 increased as the number of BCAs increased to form specific bacterial mixtures, but certain combinations of BCAs were not inhibitory to V108LRUH1. Overall, mixtures 34569 and 3456 were most inhibitory against V108LRUH1 in the guttation fluids. The effect of BCA mixtures on suppression of leaf infection was demonstrated in several plant inoculation tests, and mixtures 34569 and 3456 applied at $10^{8} \mathrm{CFU} / \mathrm{ml}$ completely suppressed leaf infection in many cases. Both in vitro and in vivo tests showed that removal of GUT9 from mixture 34569 did not have any detrimental effects on the efficacy of BCAs. This indicates that GUT9 is dispensable in the BCA mixture.

Effects of the BCAs on disease suppression were evaluated accurately using bioluminescence as a phenotypic marker for detecting sites of infection. It was shown that symptom development did not synchronize with advancement of leaf infection in certain cultivars (e.g., 'ARCS' and 'UH1060') (4) or when the pathogen entered the host tissues through wounds (1). In these cases, the actual extent of leaf infection advanced far beyond the margins of visible symptoms on the leaves. In this study, the BCAs were delivered directly to the wounds to examine interactions between the pathogen and the BCAs in the xylem and other internal leaf tissues. It was demonstrated that infected leaf tissues emitting detectable bio-

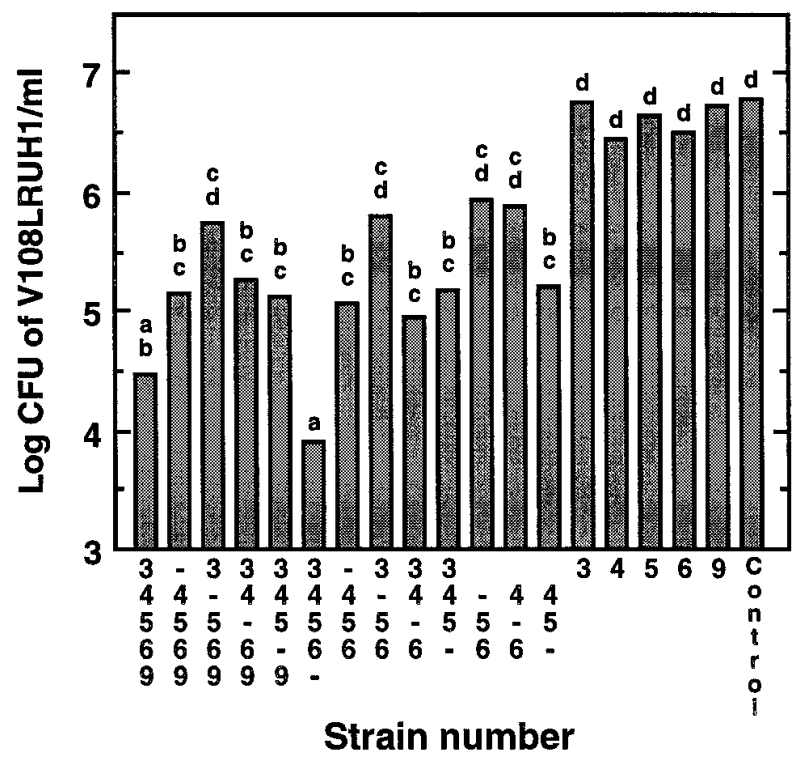

Fig. 2. Inhibitory effects of various combinations of biocontrol agents on survival of V108LRUH1 after 14 days of incubation in filter-sterilized guttation fluids of three anthurium cultivars. Cultivars were considered as replicates, and the test was done twice (three replicates per treatment in each test). Cell density of V108LRUH1 was determined 14 days after inoculation. The bars represent mean values from two repeated tests (six replicates in total). The results of three-way (strains, incubation period, and experiment) analysis of variance indicated that the effect of experiment was not significant $(P>0.05)$ and any interactions with experiment were not significant. Bars with the same letter are not significantly different $(P=0.01)$ according to the StudentNewman-Keuls test. The initial cell density of V108LRUH1 was 6.62 and $6.55 \log \mathrm{CFU} / \mathrm{ml}$ in the first and second tests, respectively. Cell densities of GUT3, GUT4, GUT5, GUT6, and GUT9 in inoculum suspensions before mixing were $7.7,8.8,8.9,8.9$, and $9.0 \log \mathrm{CFU} / \mathrm{ml}$, respectively, in the first test, and $8.5,8.7,8.8,8.8$, and $8.2 \log \mathrm{CFU} / \mathrm{ml}$, respectively, in the second test. 
luminescence contained at least $10^{6} \mathrm{CFU}$ of the pathogen per $\mathrm{cm}^{2}$ (4). In evaluating disease severity on the leaves showing little or no visible symptoms, bioluminescence was an effective method for measuring severity of leaf infection without monitoring symptom development or reisolating the pathogen from the leaf tissues by destructive sampling.

The phenomena observed in guttation fluids did not always correspond to the phenomena observed in plant inoculation tests. None of the individual strains were inhibitory to V108LRUH1 in guttation fluids, but they exhibited various levels of effectiveness in disease suppression when inoculated into the plants. Among five BCAs, GUT6 was most effective in disease suppression, yet was not inhibitory to V108LRUH1 in the guttation fluids. Likewise, mixtures 69 and 3469 were not inhibitory to V108LRUH1 in guttation fluids of 'Marian Seefurth', but they were as effective as mixture 34569 on the plant.

One possible explanation to this contradiction is that any single strain inoculated onto the plants becomes a part of the indigenous bacterial community. It was shown that all guttation fluids from individual leaves contained $\cong 10^{7} \mathrm{CFU}$ of bacteria per $\mathrm{ml}$, and the inhibitory effect of each guttation fluid was highly variable depending on the bacterial community inhabiting the fluid (3). It is noteworthy that GUT4 and GUT5 were the most important component strains among five BCAs for inhibition of V108LRUH1 in guttation fluids, and strains belonging (or closely related) to the same taxonomic groups were frequently isolated during the initial screening for BCA candidates. Assuming that the leaves are colonized by GUT4 or GUT5 (or related species), inoculation of GUT6 onto the leaves would produce the highly inhibitory mixture 456, whereas inoculation of GUT4 or GUT5 would not make any differences. Interestingly, GUT5 inoculated alone was highly effective on 'ARCS' but not on three other cultivars. It was shown that inhibitory effects of (nonsterilized) guttation fluids were considerably different among cultivars (3). These results suggest that indigenous bacterial communities may be specific to each cultivar, and thus, the effect of any single strain varies depending on the cultivar. Thus, the most effective and reliable inoculum for achieving consistent biological control over a range of cultivars would be mixture 3456 , which also was shown to be most inhibitory to V108LRUH1 in guttation fluids.
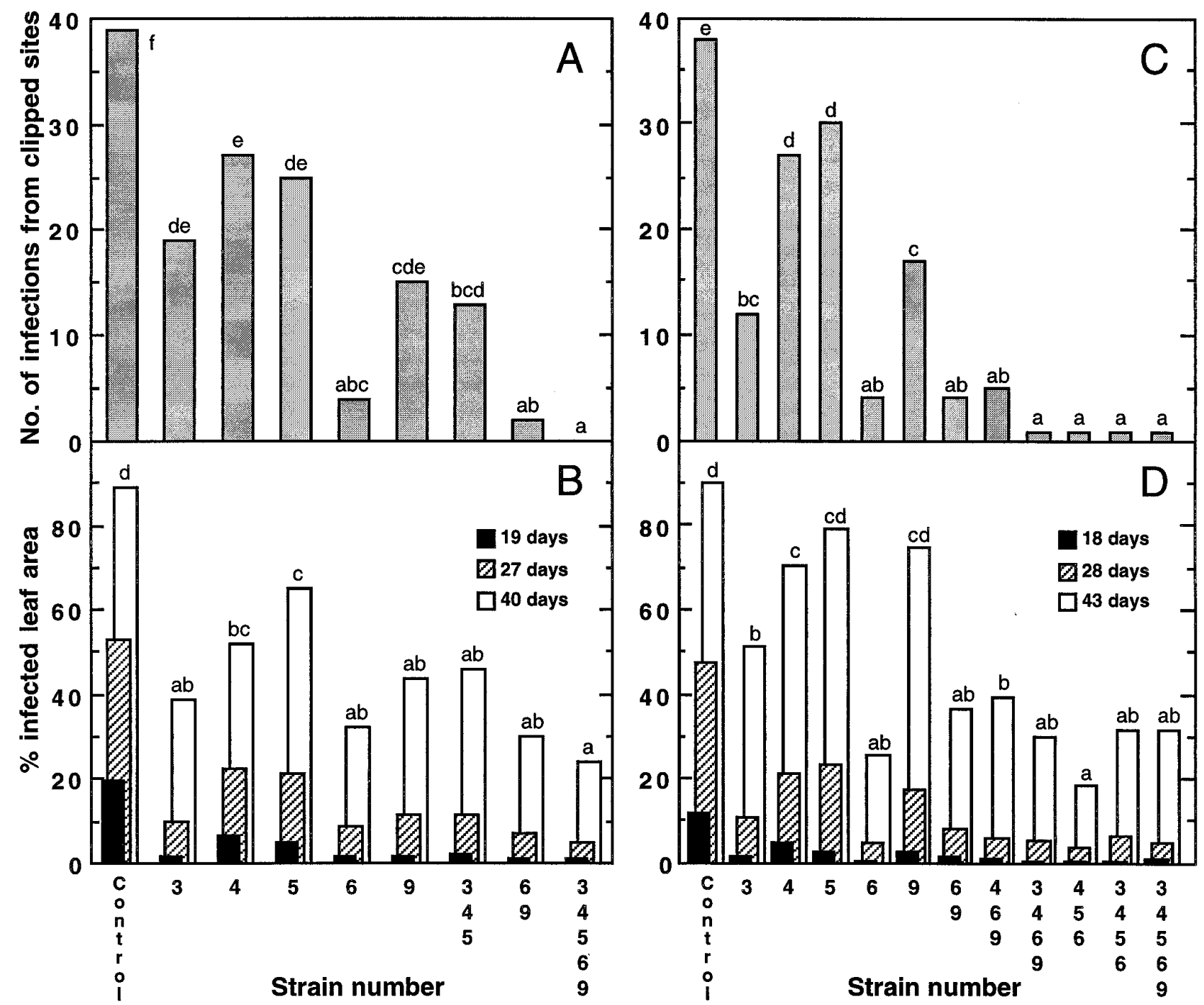

Fig. 3. Effects of individual strains and various mixtures of biocontrol agents on leaf infection by V108LRUH1. A and C, Number of sites (wounds created by clipping) invaded by the pathogenin the first and second tests, respectively. There were 40 notched sites in total: four sites per leaf and 10 leaves. Bars with the same letter are not significantly different $(P=0.01)$ according to the Student-Newman-Keuls (SNK) test. B and D, Percentage of infected leaf area determined by detecting bioluminescence in the first and second tests, respectively. Leaf infection was assessed three times with 10 observations (five plants per treatment and two leaves per plant). The treatment $\times$ assessment day interaction was significant $(P<0.001)$ in both tests. Bars designating the third assessment with the same letter are not significantly different $(P=0.01)$ according to the SNK test. The results of mean separation for the first and second assessments are not shown. Cell densities of GUT3, GUT4, GUT5, GUT6, and GUT9 in inoculum suspensions before mixing were 8.6, 8.5, 8.4, 8.1, and 8.3 log CFU/ml, respectively, in the first test, and 8.2, 8.2, 8.5, 7.9, and $8.1 \log \mathrm{CFU} / \mathrm{ml}$, respectively, in the second test. 
The essential mixture of BCAs consists of four species that have not been described as BCAs. GUT3 and GUT5 were identified as Sphingomonas paucimobilis and Brevundimonas vesicularis, respectively. GUT4 is closely related to Microbacterium imperiale. The identity of GUT6 has not yet been determined. The result of fatty acid analysis indicated $P$. rubrisubalbicans, the causal agent of mottled stripe of sugarcane (6), as the possible species. However, several plant inoculation tests on various cultivars of sugarcane confirmed that GUT6 did not cause mottled stripe (or any other symptom) in any sugarcane cultivars. The second closest species for GUT6 determined by the Biolog MicroStation system was $P$. huttiensis. More taxonomical studies are needed to confirm identities of GUT4 and GUT6.

The mixtures of BCAs were effective in all cultivars tested, indicating that biocontrol by the BCAs was not cultivar specific. More significantly, the mixtures of BCAs suppressed disease severity to nearly the same levels in six different cultivars, regardless of their susceptibility to bacterial blight. BCAs that are independent of cultivar susceptibility would be highly valuable for anthurium production, because as many as 40 cultivars are commonly produced for the market and new cultivars are being developed for a highly competitive market.

A potential advantage in biocontrol using bacterial mixtures is that the BCAs could be exchangeable. Our approach in selecting effective BCAs was to find an effective bacterial mixture first and remove trivial strains from the mixture, instead of adding strains to the most effective candidate. The results of this study indicated that even GUT3 could be removed from mixture 3456 without diminishing the efficacy of the BCAs. Rather than removing GUT3, it could be exchanged with a different strain or strains with known desirable traits for biocontrol to formulate a new improved BCA mixture.

A common criticism of biocontrol is that disease control performance by BCAs is often inconsistent under field conditions. Theoretically, mixtures of BCAs are expected to be superior to individual strains under variable field conditions, because mixtures of strains would provide stable bacterial communities with increased phenotypic diversity, expression of multiple traits for dis-

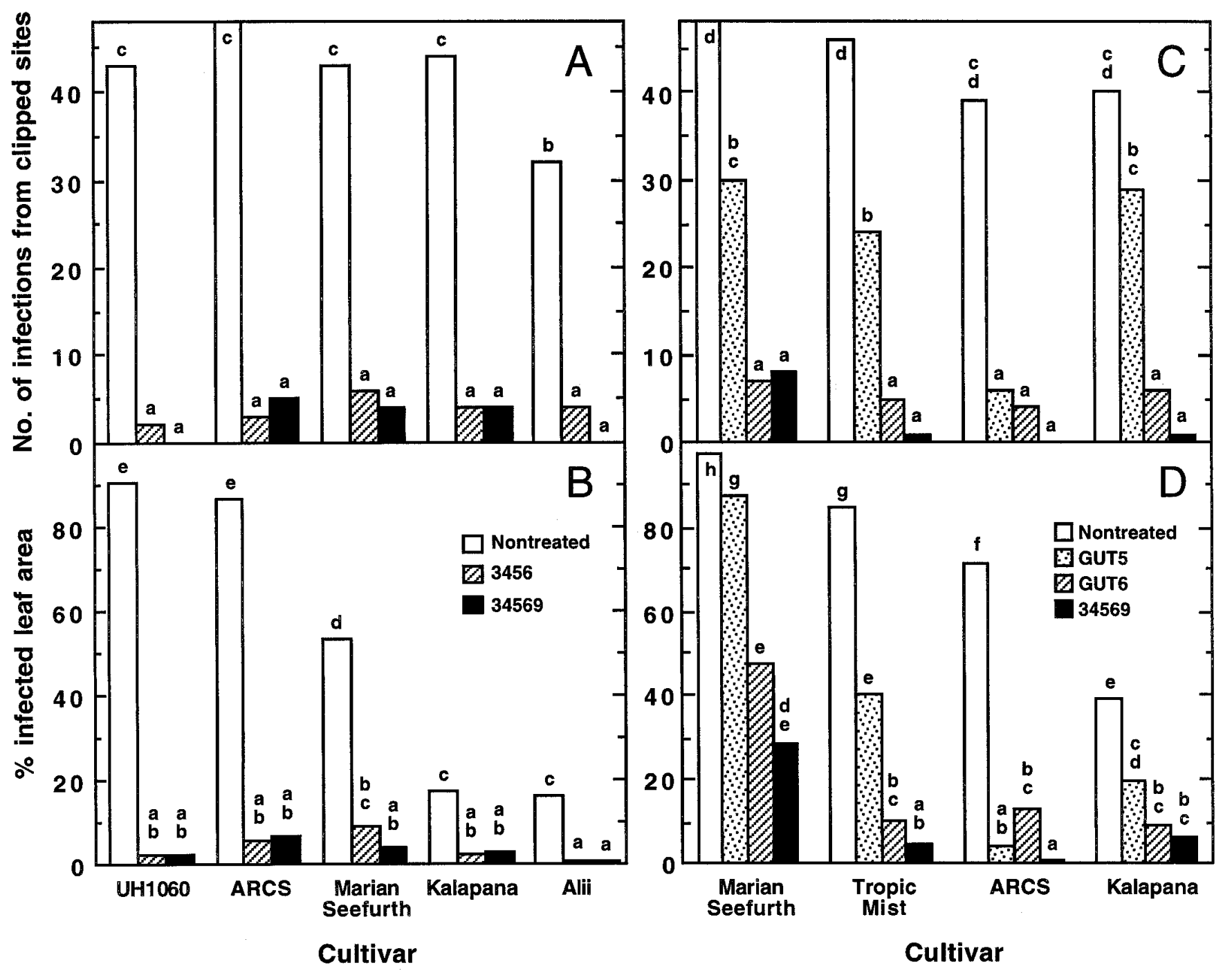

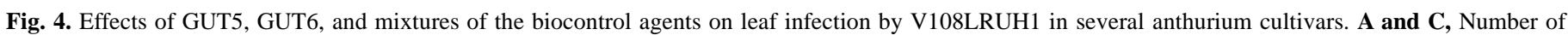

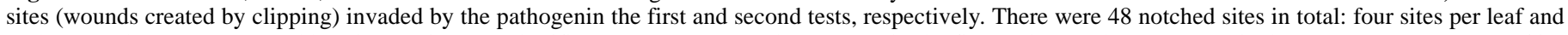

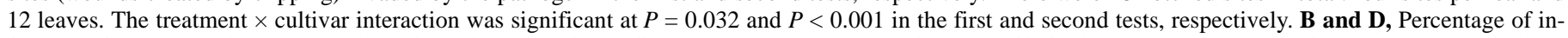

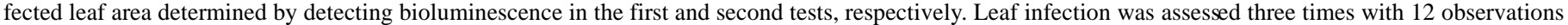

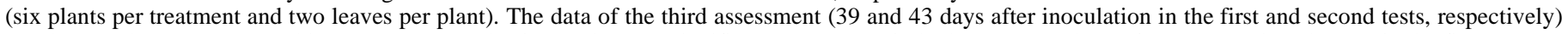

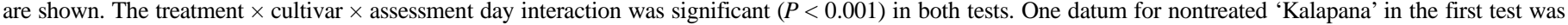

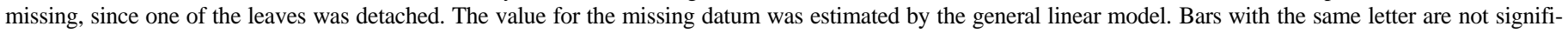

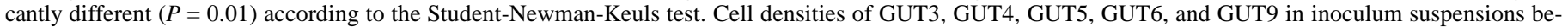

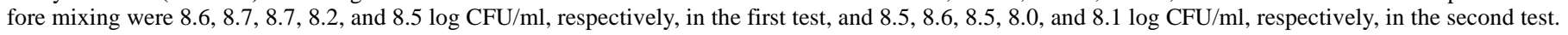


ease control, and suppression of a broad range of pathogens $(19,20)$. Thus, exchangeable BCAs would be more versatile and could be reformulated to adapt to different environmental conditions, various farming situations (e.g., pesticide application), or even different pathogens. Exchangeable BCAs may solve some of the current limitations in biocontrol.

The mixture of BCAs has promising features for effective biocontrol under field conditions. Mixture 3456 also was highly inhibitory to wild-type strains D150 and D182 of X. campestris pv. dieffenbachiae when coinoculated in the guttation fluids (R. Fukui, H. Fukui, and A. M. Alvarez, unpublished data). These two strains represent two major, distinct phenotypic populations of the pathogen isolated from commercial fields $(17,18)$. The mixtures of BCAs were highly effective in preventing wound invasion by the pathogen. This is the desirable property of BCA for controlling anthurium blight, because anthurium production involves many wound-producing practices such as vegetative propagation from plant parts, transplanting, flower harvest, defoliation, and shipping. It was also demonstrated that systemic infection initiated from infection during defoliation or flower harvest was prevented almost completely by applying mixture 3456 to cut end of petioles (H. Fukui, R. Fukui, and A. M. Alvarez, unpublished data).

Although the mixture of BCAs shows potential for effective biocontrol, more studies are needed before it is applied under field conditions. Anthurium is a long-term, perennial crop, and the climate that is favorable for anthurium production also favors the disease. Thus, the windows for infection in anthurium blight is year long. We do not expect that one-time application can control the disease sufficiently under field conditions. Most likely, the BCAs should be applied repeatedly to control the disease effectively under field conditions. The longevity of biocontrol by the mixture of BCAs needs to be studied to determine appropriate treatment intervals for field applications.

The mechanism of disease suppression for anthurium blight is not known, but nutrient competition is one likely explanation by which the pathogen is inhibited by BCAs in guttation fluids. There were significant differences in carbon utilization patterns among the strains of BCAs. Nutritional niche differentiation is known to allow different bacterial strains to coexist in a given ecosystem $(28,29)$ and improve biocontrol $(7)$. On the other hand, nearly all of the carbon sources in the Biolog system that are utilized by $X$. campestris pv. dieffenbachiae also were utilized by the BCAs as a mixture. This is probably why all five BCAs survived in guttation fluids for 2 weeks but only the pathogen was inhibited when coinoculated in guttation fluids (3). In addition, inhibition of V108LRUH1 by the BCAs was reversed by adding nutrients such as glucose, peptone, and yeast extract to guttation fluids (3). These findings suggest that nutrients could play a key role in inhibition of the pathogen in the guttation fluid. Detailed studies on effects of specific nutrients on growth and survival of BCAs and disease suppression would provide valuable information on the mechanism of biocontrol by the bacterial mixture.

\section{ACKNOWLEDGMENTS}

This research was supported by the U.S. Department of Agriculture/Special Grants Program for Tropical and Subtropical Agricultural Research (agreement no. 96-34135-2841). Journal Series No. 4400 of the Hawaii Institute of Tropical Agriculture and Human Resources. We thank A. R. Kuehnle and D. P. Schmitt for critical reading of the manuscript; S. Schenck, Hawaii Agricultural Research Center, for providing data on pathogenicity of the unidentified strain (GUT6) on various sugarcane cultivars; and A. K. Nishii and T. K. Shiraishi for their technical assistance throughout this study.

\section{LITERATURE CITED}

1. Alvarez, A., McElhaney, R., and Fukui, R. 1994. Studies of the infection process in anthurium blight using a bioluminescent strain of Xantho- monas campestris pv. dieffenbachiae. Pages 31-37 in: Proc. Hawaii Anthurium Ind. Conf., 6th. K. M. Delate and E. R. Yoshimura, eds. HITAHR J. No. 09.12.94. University of Hawaii, Honolulu.

2. Fukui, H., Alvarez, A. M., and Fukui, R. 1998. Differential susceptibility of anthurium cultivars to bacterial blight in foliar and systemic infection phases. Plant Dis. 82:800-806.

3. Fukui, R., Fukui, H., and Alvarez, A. M. 1999. Suppression of bacterial blight by a bacterial community isolated from the guttation fluids of anthuriums. Appl. Environ. Microbiol. 65:1020-1028.

4. Fukui, R., Fukui, H., McElhaney, R., Nelson, S. C., and Alvarez, A. M. 1996. Relationship between symptom development and actual sites of infection in leaves of anthurium inoculated with a bioluminescent strain of Xanthomonas campestris pv. dieffenbachiae. Appl. Environ. Microbiol. 62:1021-1028.

5. Fukui, R., Schroth, M. N., Hendson, M., and Hancock, J. G. 1994. Interactions between strains of pseudomonads in sugar beet spermospheres and their relationship to pericarp colonization by Pythium ultimum in soil. Phytopathology 84:1322-1330.

6. Hayward, A. C. 1962. Studies on bacterial pathogens of sugar cane. II. Differentiation, taxonomy and nomenclature of the bacteria causing red stripe and mottled stripe diseases. Mauritus Sugar Ind. Res. Inst. Occas. Pap. 13:13-27.

7. Janisiewicz, W. 1996. Ecological diversity, niche overlap, and coexistence of antagonists used in developing mixtures for biocontrol of postharvest diseases of apples. Phytopathology 86:473-479.

8. Janisiewicz, W., and Bors, B. 1995. Development of a microbial community of bacterial and yeast antagonists to control wound-invading postharvest pathogens of fruits. Appl. Environ. Microbiol. 61:3261-3267.

9. Kado, C. I., and Heskett, M. G. 1970. Selective media for isolation of Agrobacterium, Corynebacterium, Erwinia, Pseudomonas, and Xanthomonas. Phytopathology 60:969-976.

10. Kamemoto, H., Kuehnle, A. R., Kunisaki, J. T., Aragaki, M., Higaki, T., and Imamura, J. 1990. Breeding for bacterial blight resistance in anthurium. Pages 45-48 in: Proc. Anthurium Blight Conf., 3rd. A. M. Alvarez, ed. HITAHR J. No. 05.07.90. University of Hawaii, Honolulu.

11. Kamemoto, H., Kuehnle, A. R., Kunisaki, J. T., Aragaki, M., Higaki, T., and Imamura, J. 1991. Breeding for bacterial blight resistance in anthurium. Pages 39-41 in: Proc. Hawaii Anthurium Ind. Conf., 4th. A. M. Alvarez, D. C. Deardorff, and K. B. Wadsworth, eds. HITAHR J. No. 04.06.91. University of Hawaii, Honolulu.

12. Kamemoto, H., Kuehnle, A. R., Kunisaki, J. T., Aragaki, M., Higaki, T., and Imamura-Lichty, J. 1994. Breeding for new and bacterial blight resistance cultivars: A progress report. Pages 39-40 in: Proc. Hawaii Anthurium Ind. Conf., 5th. K. M. Delate and C. H. M. Tome, eds. HITAHR J. No. 02.02.94. University of Hawaii, Honolulu.

13. Kreig, N. R., and Holt, J. G., eds. 1984. Bergey's Manual of Systemic Bacteriology, Vol. 1. Williams and Wilkins, Baltimore, MD.

14. Mazzola, M., Fujimoto, D. K., Thomashow, S., and Cook, R. J. 1995. Variation in sensitivity of Gaeumannomyces graminis to antibiotics produced by fluorescent Pseudomonas spp. and effect on biological control of take-all of wheat. Appl. Environ. Microbiol. 61:2554-2559.

15. Nishijima, W. T. 1989. Current anthurium blight control recommendations. Pages 7-9 in: Proc. Anthurium Blight Conf., 2nd. J. A. Fernandez and W. T. Nishijima, eds. HITAHR J. No. 03.10.89. University of Hawaii, Honolulu.

16. Norman, D. J., and Alvarez, A. M. 1989. A rapid method for the presumptive identification of Xanthomonas campestris pv. dieffenbachiae and other xanthomonads. Plant Dis. 73:654-658.

17. Norman, D. J., and Alvarez, A. M. 1994. Latent infections of in vitro anthurium caused by Xanthomonas campestris pv. dieffenbachiae. Plant Cell Tissue Organ Cult. 39:55-61.

18. Norman, D. J., and Alvarez, A. M. 1994. Rapid detection of Xanthomonas campestris pv. dieffenbachiae in anthurium plants with a miniplate enrichment/ELISA system. Plant Dis. 78:954-958.

19. Pierson, E. A., and Weller, D. M. 1994. Use of mixtures of fluorescent pseudomonads to suppress take-all and improve the growth of wheat. Phytopathology 84:940-947.

20. Raupach, G. S., and Kloepper, J. W. 1998. Mixtures of plant growthpromoting rhizobacteria enhance biological control of multiple cucumber pathogens. Phytopathology 88:1158-1164.

21. Sathyanarayana, N., Reddy, O. R., and Rajak, R. L. 1998. Interception of Xanthomonas campestris pv. dieffenbachiae on anthurium plants from the Netherlands. Plant Dis. 82:262.

22. Schisler, D. A., Slininger, P. J., and Bothast, R. J. 1997. Effects of antagonist cell concentration and two-strain mixtures on biological control of Fusarium dry rot of potatoes. Phytopathology 87:177-183.

23. Segers, P., Vancanneyt, M., and Pot, B. 1994. Classification of Pseudomonas diminuta Leifson and Hugh 1954 and Pseudomonas vesicularis Busing, Doll, and Freytag 1953 in Brevundimonas gen. nov. as Brevundimonas diminuta comb. nov. and Brevundimonas vesicularis comb. 
nov., respectively. Int. J. Syst. Bacteriol. 44:499-510.

24. Shaw, J. J., and C. I. Kado. 1987. Direct analysis of the invasiveness of Xanthomonas campestris mutants generated by $\mathrm{Tn} 4431$, a transposon containing a promoterless luciferase cassette for monitoring gene expression. Pages 57-60 in: Molecular Genetics of Plant-Microbe Interactions. D. P. S. Verma and N. Nelson, eds. Martinus Nijhoff, Boston.

25. Sivasithamparam, K., and Parker, C. A. 1978. Effects of certain isolates of bacteria and actinomycetes on Gaeumannomyces graminis var. tritici and take-all of wheat. Aust. J. Bot. 26:773-782.

26. Sneath, P. H., and Holt, J. G., eds. 1984. Bergey's Manual of Systemic Bacteriology, Vol. 2. Williams and Wilkins, Baltimore, MD.

27. Wilson, E. E., Zeitoun, F. M., and Fredrickson, D. L. 1967. Bacterial phloem canker, a new disease of Persian walnut trees. Phytopa- thology 57:618-621.

28. Wilson, M., and Lindow, S. E. 1994. Ecological similarity and coexistence of epiphytic ice-nucleating $\left(\right.$ Ice $\left.^{+}\right)$Pseudomonas syringae strains and a non-ice-nucleating (Ice $\left.{ }^{-}\right)$biological control agent. Appl. Environ. Microbiol. 60:3128-3137.

29. Wilson, M., and Lindow, S. E. 1994. Coexistence among epiphytic bacterial populations mediated through nutritional resource partitioning. Appl. Environ. Microbiol. 60:4468-4477.

30. Yabuuchi, E., Yano, I., and Oyaizu, H. 1990. Proposals of Sphingomonas paucimobilis gen. nov. and comb. nov. Sphingomonas parapaucimobilis sp. nov., Sphingomonas yanoikuyae sp. nov., Sphingomonas adhaesiva sp. nov., Sphingomonas capsulata comb. nov. and two genospecies of the genus Sphingomonas. Microbiol. Immunol. 34:99-119. 